\title{
miR-23a impairs bone differentiation in osteosarcoma via down-regulation of GJA1
}

\section{OPEN ACCESS}

Edited by:

David Azorsa,

Ron Matricaria Institute of Molecular Medicine at Phoenix Children's

Hospital, USA

Reviewed by:

Jian-Jun Wei,

Northwestern University, USA

Lihua $L i$,

University of Minnesota, USA

*Correspondence:

Paul S. Meltzer,

Genetics Branch, Center for Cancer Research, 37 Convent Drive, Room 6144, Bethesda, MD 20892, USA

pmeltzer@mail.nih.gov

${ }^{\dagger}$ Present Address: Yevgeniy Gindin,

The Genome Institute, Washington University School of Medicine,

Saint Louis, USA Princy Francis,

Mayo Clinic, Scottsdale, USA Ogan D. Abaan,

Lockheed Martin IS\&GS Civil, Rockville, USA

${ }^{\ddagger}$ These authors have contributed equally to this work.

Specialty section: This article was submitted to Cancer Genetics,

a section of the journal

Frontiers in Genetics

Received: 13 April 2015 Accepted: 18 June 2015

Published: 02 July 2015

Citation: Gindin $Y$, Jiang $Y$, Francis $P$, Walker RL, Abaan OD, Zhu YJ and Meltzer

PS (2015) miR-23a impairs bone differentiation in osteosarcoma via down-regulation of GJA1.

Front. Genet. 6:233.

doi: 10.3389/fgene.2015.00233

\author{
Yevgeniy Gindin ${ }^{1,2 \dagger \neq}$, Yuan Jiang ${ }^{1 \neq}$, Princy Francis ${ }^{1 \dagger}$, Robert L. Walker ${ }^{1}$, Ogan D. Abaan ${ }^{1 \dagger}$, \\ Yuelin J. Zhu' ${ }^{1}$ and Paul S. Meltzer ${ }^{\text {* }}$ \\ ${ }^{1}$ Genetics Branch, Center for Cancer Research, National Institutes of Health, Bethesda, MD, USA, ${ }^{2}$ Graduate Program in \\ Bioinformatics, Boston University, Boston, MA, USA
}

Osteosarcoma is the most common type of bone cancer in children and adolescents. Impaired differentiation of osteoblast cells is a distinguishing feature of this aggressive disease. As improvements in survival outcomes have largely plateaued, better understanding of the bone differentiation program may provide new treatment approaches. The miRNA cluster miR-23a 27a 24-2, particularly miR-23a, has been shown to interact with genes important for bone development. However, global changes in gene expression associated with functional gain of this cluster have not been fully explored. To better understand the relationship between miR-23a expression and bone cell differentiation, we carried out a large-scale gene expression analysis in HOS cells. Experimental results demonstrate that over-expression of miR-23a delays differentiation in this system. Downstream bioinformatic analysis identified miR-23a target gene connexin-43 (Cx43/GJA1), a mediator of intercellular signaling critical to osteoblast development, as acutely affected by miR-23a levels. Connexin-43 is up-regulated in the course of HOS cell differentiation and is down-regulated in cells transfected with miR-23a. Analysis of gene expression data, housed at Gene Expression Omnibus, reveals that Cx43 is consistently up-regulated during osteoblast differentiation. Suppression of Cx43 mRNA by miR-23a was confirmed in vitro using a luciferase reporter assay. This work demonstrates novel interactions between microRNA expression, intercellular signaling and bone differentiation in osteosarcoma.

Keywords: miR-23a, GJA1, bone, differentiation, osteosarcoma

\section{Introduction}

Osteosarcoma is the most common primary bone malignancy and occurs most frequently in adolescents (Mirabello et al., 2009). Osteosarcoma tumors most often arise in the long bones of the skeleton, with more than half presenting around the knee (Broadhead et al., 2011), and is less common in axial skeleton (Martin et al., 2012). At diagnosis, 20\% of osteosarcoma patients present with lung metastases with an additional $40 \%$ developing metastases at later stage (Martin et al., 2012). Survival rates for localized osteosarcoma are at 60-70\% (Longhi et al., 2006; Mirabello et al., 2009), while the 5-year survival for osteosarcoma patients with metastases is $20 \%$ (PosthumaDeBoer et al., 2011). Despite intense research efforts, the survival rates for osteosarcoma have remained essentially unchanged for over two decades (Longhi et al., 2006; Mirabello et al., 2009). Contributing to the challenge of understanding and ultimately developing 
effective treatments for osteosarcoma is its complex karyotype and high level of chromosomal instability (Helman and Meltzer, 2003). With the increasing understanding of osteosarcoma biology, perturbation of cell differentiation is often regarded as aspect of this disease (Thomas and Kansara, 2006; Tang et al., 2008). Therefore, it is possible that the path toward developing new treatment approaches for osteosarcoma lies through an improved understanding of the dysregulation of the bone differentiation program in this devastating disease.

Discovery of small (about 22nt in length) non-coding RNA species, termed microRNAs (miRNAs), has, in many ways, revolutionized the understanding of gene expression regulation. It is now recognized, for instance, that miRNAs contribute to many biological processes (Ambros, 2004) and that their expression patterns can be used to classify cancers ( $\mathrm{Lu}$ et al., 2005; Bloomston et al., 2007), suggesting that miRNAs play the roles similar to tumor suppressors and oncogenes (Dalmay and Edwards, 2006; Esquela-Kerscher and Slack, 2006). MicroRNAs play an integral role in controlling cell differentiation by suppressing genes that maintain plasticity (Yi et al., 2008), or by suppressing genes that inhibit cell-lineage commitment ( $\mathrm{Li}$ et al., 2008) or through a combination of the two (Forrest et al., 2010).

miRNAs play a paramount role in bone differentiation (Sugatani and Hruska, 2007; Kobayashi et al., 2008; Wang et al., 2008; Inose et al., 2009; Hassan et al., 2012). Recent studies identified miRNA biomarkers relevant to therapy response and identification of therapeutic targets (Lulla et al., 2011; Maire et al., 2011; Jones et al., 2012; Cai et al., 2013). Much attention has been devoted to the role of miR-23a in bone differentiation, primarily via its targeting (both direct and indirect) of transcription factors essential to osteoblastogenesis such as TRPS1, RUNX2, and SATB2 (Hassan et al., 2010; Zhang et al., 2011, 2012). In the current work, we study the effects of miR-23a expression in HOS cells, which are distinguished from other human osteosarcoma cells by their ability to undergo a bone cell lineage differentiation program (Siggelkow et al., 1998; Hassan et al., 2010).

\section{Materials and Methods}

\subsection{Cell Culture and Bone Differentiation}

All cell lines were obtained from ATCC. The cells were grown in DMEM media with $10 \%$ fetal bovine serum and supplemented with $1 \%$ penicillin and streptomycin. HOS cells were grown to got $100 \%$ confluence, followed by differentiation at 7-9 days induced by bone inducing agents, that include $\mathrm{L}$-ascorbic acid $50 \mathrm{ug} / \mathrm{ml}$ and beta-glycerophosphate $5 \mathrm{mM}$ (Hassan et al., 2006). Cells were harvested at indicated times for mRNA and protein extraction or fixed with $10 \%$ neutral-buffered formalin (NBF) for detection of calcium deposits by Alizarin Red staining.

\subsection{RNA Analyses}

Total RNA was isolated using Trizol reagent (Invitrogen), treated with DNase I (Ambion) and reverse transcribed using "iScript Reverse Transcription Supermix for RT-qPCR" (BIORAD). GJA1 and COL1A1 gene expression qRT-PCR were performed using the TaqMan Gene Expression Assays (ABI/ Life Technologies). mRNA levels were normalized to housekeeping gene ACTB. miRNA-23a was quantified in triplicate using the TaqMan MicroRNA Assay (ABI/ life technologies) and normalized to U6. mRNA levels were assayed for relative expression using procedure described in Livak and Schmittgen (2001).

\subsection{Illumina Expression Arrays}

HOS cells were transfected with human hsa-miR-23a, or negative control mimic (Thermo Scientific) for $72 \mathrm{~h} .150 \mathrm{ng}$ of each RNA was amplified and labeled using the "Illumina TotalPrep RNA Amplification kit" (LifeTechnologies). The biotin-labeled cRNAs were quantitated spectrophotometrically and $750 \mathrm{ng}$ was hybridized to Illumina HumanRef-8v3 Expression BeadChip microarrays (Illumina, San Diego, CA). BeadChips were scanned in an Illumina Scanner. The data has been deposited in the GEO database under accession number. One SuperSeries record GSE68014; Two regular Series records: GSE68012 and GSE68012.

\subsection{Protein Immunoblot Analyses}

Whole cell lysates from transfected HOS cells were prepared using RIPA buffer. Proteins were analyzed by SDS PAGE, transferred to nitrocellulose membranes and probed with GJA1 antibody (ab11370 Abcam). Western Blots were quantified by densitometry.

\subsection{Luciferase Reporter Assay}

HOS cells were co-transfected in 24 well-plates using Lipofectamine 2000 (Invitrogen) with $20 \mathrm{nM}$ miR-23a mimic or control miRNA mimic and $100 \mathrm{ng}$ of psiCHECK2- 3UTR (Promega) vector containing the GJA1-3UTR cloned into the multiple cloning site of Renilla luciferase. After $48 \mathrm{~h}$ of transfection luciferase activity was measured using the Dual Luciferase Assay System (Promega). The experiment was performed in triplicate. Results were normalized to those obtained in cells transfected with an empty vector. Data were normalized to Firefly luciferase and results from 3 independent experiments were compared. GJA1 sequences were cloned into psiCHECK-2 by annealing complementary oligomers matching each GJA1 sequence with overhanging ends complementary to the XhoI and NotI sites of psiCHECK-2.

\section{6. siGJA1 Transfection Assay}

HOS cells were differentiated as described above. One day after induction of differentiation, cells were transfected using Lipofectamine RNAiMAX Reagent (Invitrogen) with ONTARGETplus-siGJA1-pool, siGJA1-05, siGJA1-06 (Thermo Scientific L-011042-00-0005) at a final concentration of 100 pmol. After $72 \mathrm{~h}$ transfection, on differentiation day 4 , the cells were harvested for mRNA, protein assays, and ALP activity assay or fixed with $10 \% \mathrm{NBF}$ for detection of calcium deposits by Alizarin Red Staining.

\subsection{ALP assay in siGJA1-Transfected HOS Cells}

Alkaline phosphatase activity was determined in HOS cell lysates using the colorimetric Alkaline Phosphatase Assay Kit (Abcam, Cat No: ab83369). The kit uses p-nitrophenyl phosphate as a phosphatase substrate, which turns yellow when 
dephosphorylated by alkaline phosphatase. The absorbance at $405 \mathrm{~nm}$ was measured using a multi well plate reader (550 Microplate Reader; Bio-Rad Laboratories). Each assay condition was carried out in triplicate. Cell lysates were analyzed for protein content using the Bio-Rad DC Protein Assay (Bio-Rad Laboratories), and alkaline phosphatase activity was normalized for total protein concentration.

\subsection{Alizarin Red Staining in siGJA1 Transfected HOS Cells}

HOS cells were fixed with $10 \% \operatorname{NBF}^{\star}(10 \%$ Formalin solution, neutral buffered, SIGMA HT501128-4L) on differentiation day 4 with GJA1 silencing $72 \mathrm{~h}$, followed by "Alizarin Red S Staining” (SIGMA A5533-25G) using NovaUltra Special Stain Kits protocol. The red staining is indicative of calcium deposits.

\subsection{Data Analysis}

All statistical analyses were carried out using the $\mathrm{R}$ statistical environment version 3.0. Microarray data were analyzed using limma package (Smyth, 2005). Data from GEO were obtained using the GEOquery package (Davis and Meltzer, 2007).

\section{Results}

\subsection{Induction of Differentiation in Osteosarcoma Cells}

To confirm that HOS cells are amendable to bone differentiation induction (Siggelkow et al., 1998), we treated these cells with L-ascorbic acid, which induces the formation of collagenous extracellular matrix and brings an osteoblast-specific gene expression program in osteogenic lineage cells (Franceschi et al., 1994). We then monitored HOS cell culture for the presence of calcium deposits, which serve as a marker of bone mineralization, via Alizarin Red staining. Our results indicate that HOS cells undergo osteoblast-like differentiation upon stimulation with L-ascorbic acid. HOS cells exhibit intense Alizarin Red staining on day 7 post differentiation induction (Figure 1). We confirmed this result by monitoring the expression of collagen Ia1 (COL1A1) - a gene marker of bone differentiation (Figure 2). We have observed a two-fold increase in COL1A1 mRNA levels between the initial and terminal differentiation time-points.

\section{2. miR-23a Targets Genes Involved in Bone Differentiation}

To study the effect of miR-23a on the gene expression program in osteosarcoma, we set out to identify genes that are transcriptionally repressed by miR-23a in HOS cells. To that end, we first transfected HOS cells with a miR-23a mimic and compared their gene expression profile with mock-transfected cells using Illumina microarrays. Our analysis shows that 1530 genes (see Supplemental Table 1) are down-regulated in miR23a transfected cells vs. mock-transfected cells. These genes are significantly enriched for predicted (Lewis et al., 2005) miR23a targets (262 overlapping genes (see Supplemental Table 2); $\left.P_{\text {overlap }}=4.96 \times 10^{-46}\right)$.

Having established the on-target effects of miR-23a overexpression in HOS cells, we next asked if the genes affected
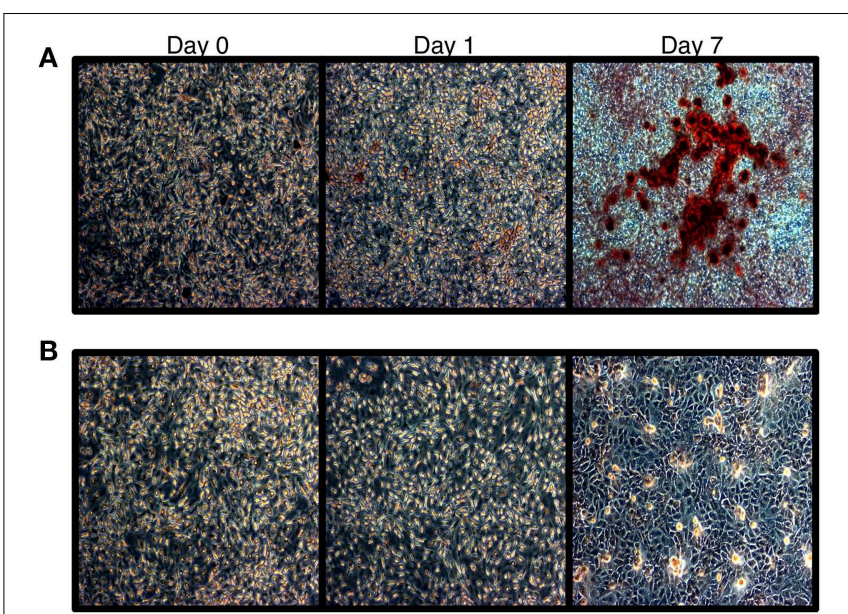

FIGURE 1 | Alizarin red staining of HOS cells. (A) Alizarin red staining of HOS cells during the differentiation time course. Red staining is indicative of calcium deposits. (B) Untreated cells.

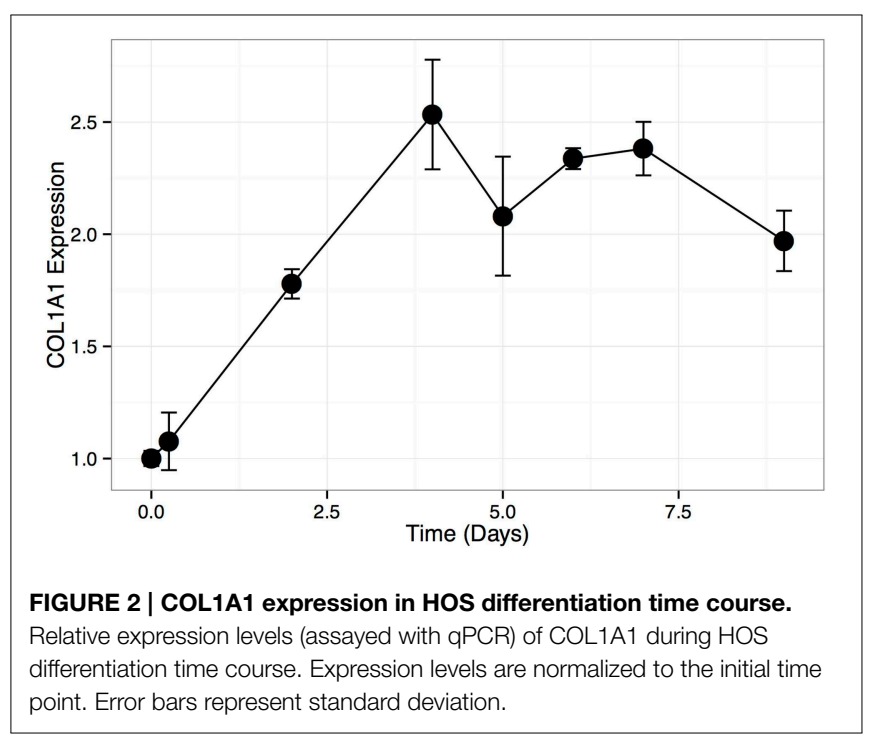

by miR-23a overlap significantly with genes that change in expression during HOS differentiation. Given miR-23a's role as a dampener of bone differentiation gene expression program (Hassan et al., 2010), we focused on genes that are upregulated during the HOS differentiation time course. To that end, we compared mRNA expression levels in HOS cells prior to differentiation induction with that of HOS cells that display phenotypic properties of bone cells post differentiation induction using microarrays in triplicate. Our analysis shows that 3065 genes (see Supplemental Table 3) increase in expression during HOS cell differentiation. Of those, 466 genes (see Supplemental Table 4) are down-regulated upon miR-23a transfection $\left(P_{\text {overlap }}=1.21 \times 10^{-10}\right)$. To identify genes of interest that are under miR-23a control and are relevant to HOS cell differentiation, we identified 77 genes (see Supplemental Table 5) that meet the following criteria: (i) are computationally predicted miR-23a targets; (ii) are down- regulated on miR-23a 
over-expression and; (iii) are up-regulated during HOS cell differentiation time course.

Transcription factor binding site enrichment analysis (Loots et al., 2002) reveals that more than one-half of the 77 genes contain an SP1 transcription factor motif within $2 \mathrm{~kb}$ of their transcription start site [42 genes (see Supplemental Table 6); $\left.P_{\text {enrichment }}=1 \times 10^{-23}\right]$. These results suggest that overexpression of miR-23a interferes with the bone differentiation program by counteracting the action of osteoblast lineage inducing transcription factors binding to the SP1 motif. The SP1 transcription factor family include osterix (SP7), a bone lineage specific factor required for osteoblast differentiation and bone formation (Nakashima et al., 2002). These results suggest that over-expression of miR-23a interferes with the bone differentiation program by counteracting the action of osteoblast lineage inducing transcription factor SP1. In order to narrow down the list of likely miR-23a targets that are involved in bone differentiation we looked for gene signature enrichments, as curated in Molecular Signatures Database (Subramanian et al., 2005), among the 42 high-quality miR23a down-regulated genes with an upstream SP1 binding site. The top-enriched signature is that of genes up-regulated upon EZH2 knockdown in prostate cancer cells (Nuytten et al., 2008) (Table 1; $\left.P_{\text {overlap }}=3.61 \times 10^{-9}\right)$ EZH2, the catalytic subunit of the PRC2 repressive complex, is commonly associated with silencing of pro-differentiation genes (Simon and Lange, 2008), a function analogous with that of miR-23a in bone differentiation. This observation is particularly interesting as prostate metastatic tumors are often osteoblastic (Logothetis and Lin, 2005) and phosphorylation of EZH2 by CDK1 is critical for osteogenic differentiation of human bone-marrow-derived mesenchymal cells (Wei et al., 2011).

\subsection{GJA1 is a Major Target of miR-23a}

In order to identify a specific miR-23a target related to bone differentiation, we examined the gene list in Table $\mathbf{1}$ for genes involved in sensing extracellular environment and intercellular communication, which are essential in bone formation that are also present in the aforementioned gene signatures. Expert-based examination of the gene list, through the use of manual curation and gene ontology functionality related to bone differentiation, led a gene whose product is connexin $(\mathrm{Cx})-43$ (also known as

\begin{tabular}{ll} 
TABLE 1 | miR-23a Target Genes Relevant to HOS Differentiation. \\
\hline Gene symbol & Gene name \\
\hline CAB39 & Calcium binding protein 39 \\
CLDN12 & Claudin 12 \\
DCBLD2 & Discoidin, CUB and LCCL domain containing 2 \\
FAM46A & Family with sequence similarity 46, member A \\
GJA1 & Gap junction protein, alpha 1, 43kDa (connexin 43) \\
IRF1 & Interferon regulatory factor 1 \\
MARCKS & Myristoylated alanine-rich protein kinase C substrate \\
RAB8B & RAB8B, member RAS oncogene family \\
TNFAIP3 & Tumor necrosis factor, alpha-induced protein 3 \\
UBL3 & Ubiquitin-like 3
\end{tabular}

GJA1). GJA1 is a member of the gap junction family and is the most abundant gap junction expressed in bone (Loiselle et al., 2013), where it facilitates response to extracellular mechanical (Jiang et al., 2007), pharmacologic and hormonal stimuli (Plotkin and Bellido, 2013) and is required for signal transduction among bone lineage cells (Civitelli, 2008). Crucially, GJA1 is essential for osteoblast differentiation in humans and animals in vivo (Stains and Civitelli, 2005a).

We verified that the miR-23a binding site is well-conserved in $3^{\prime}$ UTR of the GJA1 gene (Lewis et al., 2005; Friedman et al., 2009). We next sought to verify miR23a:GJA1 interaction in vitro. To that end we carried out a reporter assay where the 3'UTR of GJA1 was cloned into the $3^{\prime}$ UTR of a luciferase gene. We find that miR-23a significantly reduces luciferase GJA1 reporter activity (Figure 3). These results confirm that GJA1 is a bona fide miR-23a target.

We then set out to elucidate the expression pattern of GJA1 during osteoblast cell differentiation. To that end, we identified, in Gene Expression Omnibus (GEO) (Barrett et al., 2013), a dataset used in two recently published studies (Nabavi et al., 2012; Pustylnik et al., 2013) that assayed gene expression in mouse MC3T3-E1 osteoblast cells following differentiation induction by L-ascorbic acid. Neither study explicitly addressed gap junction expression in osteoblast differentiation. Our analysis of the data deposited in GEO shows that GJA1 expression increases over 500fold (6-probe average; $P<1.0 \times 10^{-6}$ ) in mouse osteoblast cells following differentiation.

Next, we asked whether GJA1 expression levels increase during HOS cell differentiation and how this expression pattern may be related to miR-23a expression. To that end, we induced differentiation in HOS cells and analyzed mRNA expression with quantitative (q)PCR. Our results show (Figure 4) that GJA1 levels increase as HOS cells begin to display phenotypical hallmarks of osteblast cells. GJA1 levels reach their maximum on day 7 post differentiation induction, at which point HOS cells display

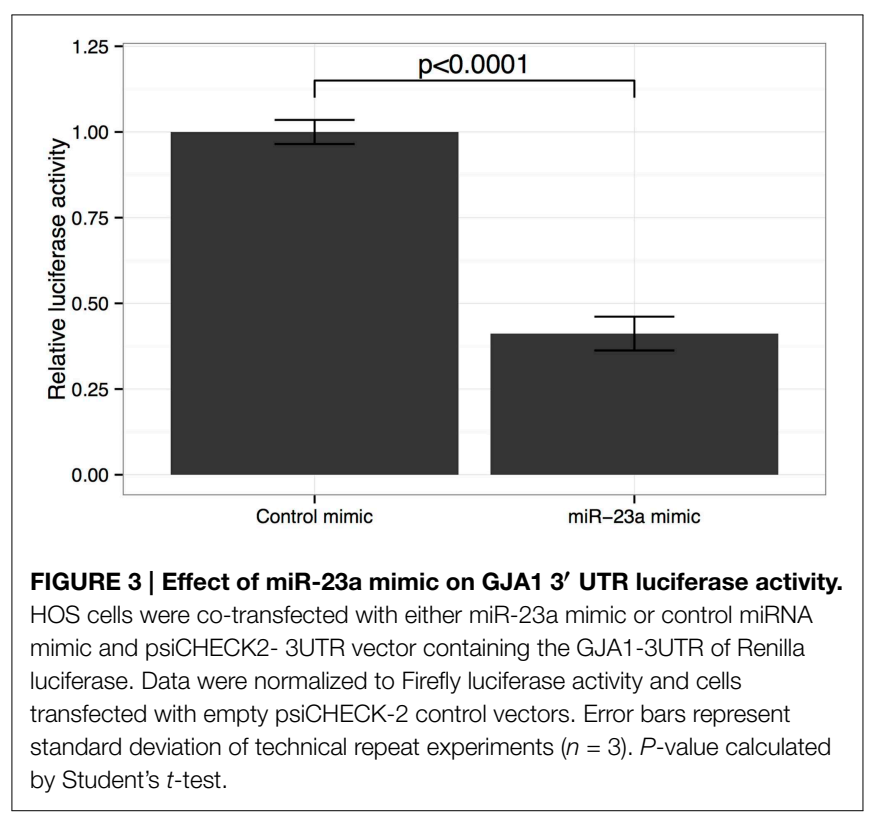


the phenotypic properties of bone differentiation (Figure 1) and COL1A1 levels have reached their peak (Figure 2). Importantly, miR-23a levels are inversely related to GJA1 expression: reaching minimum, when GJA1 levels are at their peak and Alizarin red staining is at its maximum; then increasing gradually past day 4 when GJA1 levels off and begins to decrease.

\subsection{Knock-down of GJA1 Delays HOS Cell Differentiation}

We next examined the relationship between GJA1 expression and HOS cell differentiation (Figure 5). To that end, we

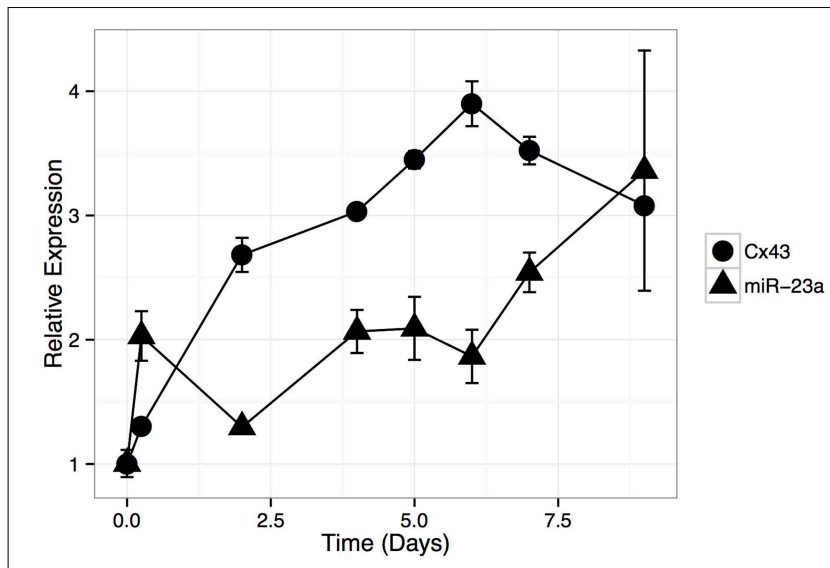

FIGURE 4 | Relative expression of GJA1 and miR-23a during HOS cell differentiation time course. HOS cells were induced to differentiate and mRNA aliquots were isolated at selected time points ( $x$-axis). Relative expression of GJA1 and miR-23a were normalized, separately, to their basal levels (Day 0). Error bars represent standard deviation due to technical repeats $(n=3)$. once again induced HOS cell differentiation, but this time transfecting HOS cells with GJA1 siRNA constructs $24 \mathrm{~h}$ after differentiation induction. First, we examined the GJA1 knock-down effect on differentiation qualitatively via Alizarin Red staining (Figure 5A). As shown in Figure 5A, cells that were transfected with GJA1 siRNA produced less extracellular calcium deposits (as evidence by stain intensity) compared to either to either of the negative controls. Lastly, we sought to quantitatively measure the effect of GJA1 knock-down on HOS cell differentiation. To that end we measured alkaline phosphatase (ALP) activity in these cells after differentiation induction and transfection with either GJA1 siRNA or negative controls. As shown in Figure 5B, there is a more than a twofold reduction in ALP activity in HOS cells where GJA1 is knocked-down in siRNA. Importantly, GJA1 siRNA transfection led to decreased GJA1 protein levels in both of these experiments (Figure 5). Together, these results demonstrate that reduction of GJA1 expression prevents HOS cells from displaying matrix calcification phenotype and ALP activity that is associated with osteoblast cells (Figure 5).

\section{Discussion}

A number of recent studies have identified miRNAs that are differentially expressed between normal bone and osteosarcoma (see recent summaries in Miao et al., 2013 and Zhou et al., 2013). Importantly, miR-23a has been shown to control bone differentiation (Hassan et al., 2010; Zhang et al., 2011, 2012). However, it is not clear what role, if any, miR-23a has within the realm of osteosarcoma. Studies that focused on the function of miR-23a in bone differentiation have been restricted to the examination of miR-23a and expression of transcription factors that are paramount to bone biology such as RUNX2 (Zhang

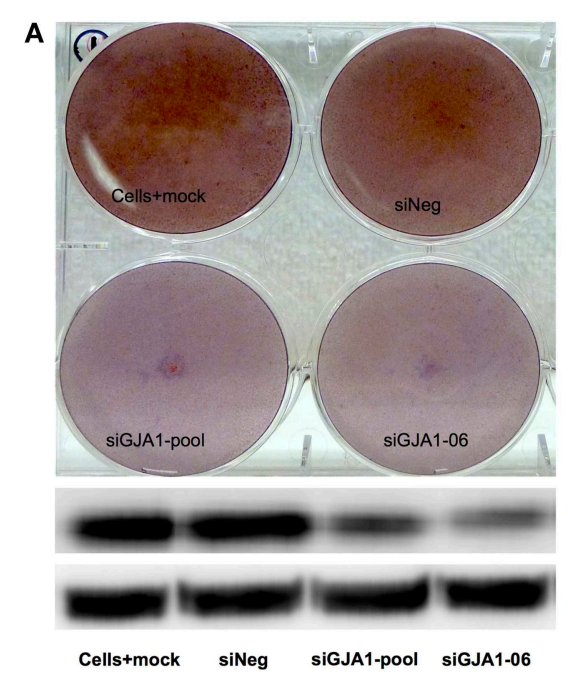

FIGURE 5 | Effect of GJA1 knock-down on HOS cell differentiation. HOS cells were induced to differentiate and were then transfected with either: (i) a scrambled siRNA negative control, or (ii) empty buffer negative control, or (iii) a pool of GJA1 siRNA constructs, or (iv) a single GJA1 siRNA construct number $6.72 \mathrm{~h}$ after transfection cells were either (A)

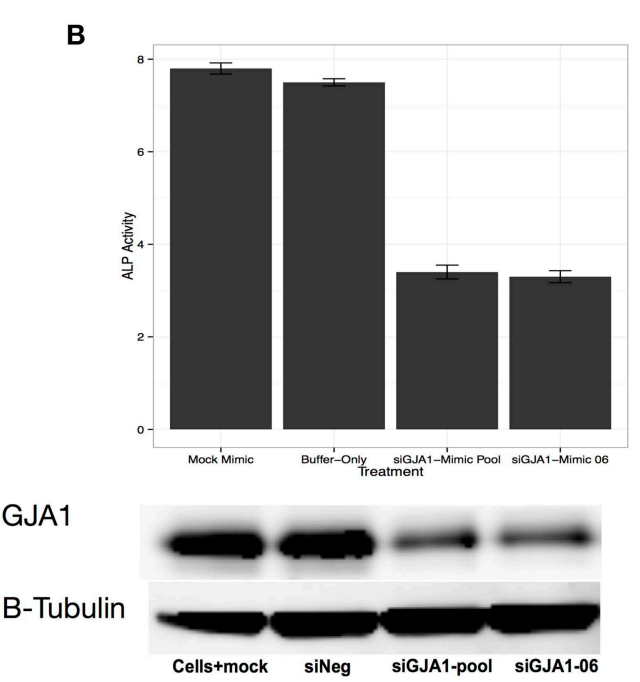

stained with Alizarin Red, or (B) subjected to measurement of alkaline phosphatase (ALP) activity. In both cases, an aliquot of cells was used to measure to GJA1 protein concentration via Western blot (bottom panels images). Error bars represent standard error from the mean from three replicates. 
et al., 2011, 2012) and/or SATB2 (Hassan et al., 2010), which were chosen a priori. We find that miR-23a acts to inhibit differentiation, at least in part, by blocking the expression of connexin 43 (GJA1) a key protein for of cell-cell communication and osteoblast differentiation.

In this study we examined the relationships between miR$23 \mathrm{a}$ and bone differentiation within the context of osteosarcoma. Previous studies demonstrated the relationship between miR-23a and transcription factors that are central to bone differentiation program (Hassan et al., 2010; Zhang et al., 2012). Separately, Inose et al. (2009) have shown that bone differentiation is negatively impacted in mice by miR-206-mediated silencing of GJA1. We could not detect miR-206 expression in HOS nor human osteoblast cells (data not shown). This points to redundant pathways that fine-tune GJA1 expression during bone differentiation.

Loss of gap junctional communication delays osteoblast differentiation and reduces the ability of these cells to form mineralized extracellular matrix (Lecanda et al., 1998; Schiller et al., 2001). A clue as to how this effect arises came from an observation that loss of GJA1 function is accompanied by diminished extracellular-signal-regulated kinase (ERK) activity (Stains and Civitelli, 2005b). In the proposed mechanism, a GJA1 gap junction allows for passage of a 2nd messenger activating ERK/PI3K signaling cascades that would in turn recruit transactivator SP1 to promoter regions of genes associated with the osteoblastic gene expression program, such as osteocalcin

\section{References}

Ambros, V. (2004). The functions of animal microRNAs. Nature 431, 350-355. doi: 10.1038/nature02871

Barrett, T., Wilhite, S. E., Ledoux, P., Evangelista, C., Kim, I. F., Tomashevsky, M., et al. (2013). NCBI GEO: archive for functional genomics data sets-update. Nucleic Acids Res. 41, D991-D995. doi: 10.1093/nar/ gks1193

Bloomston, M., Frankel, W. L., Petrocca, F., Volinia, S., Alder, H., Hagan, J. P., et al. (2007). MicroRNA expression patterns to differentiate pancreatic adenocarcinoma from normal pancreas and chronic pancreatitis. J. Am. Med. Assoc. 297, 1901-1908. doi: 10.1001/jama.297.17.1901

Broadhead, M. L., Clark, J. C. M., Myers, D. E., Dass, C. R., and Choong, P. F. M. (2011). The molecular pathogenesis of osteosarcoma: a review. Sarcoma 2011:959248. doi: 10.1155/2011/959248

Cai, H., Lin, L., Cai, H., Tang, M., and Wang, Z. (2013). Prognostic evaluation of microRNA-210 expression in pediatric osteosarcoma. Med. Oncol. 30:499. doi: $10.1007 / \mathrm{s} 12032-013-0499-6$

Civitelli, R. (2008). Cell-cell communication in the osteoblast/osteocyte lineage. Arch. Biochem. Biophys. 473, 188-192. doi: 10.1016/j.abb.2008.04.005

Dalmay, T., and Edwards, D. R. (2006). MicroRNAs and the hallmarks of cancer. Oncogene 25, 6170-6175. doi: 10.1038/sj.onc.1209911

Davis, S., and Meltzer, P. S. (2007). GEOquery: a bridge between the Gene Expression Omnibus (GEO) and BioConductor. Bioinformatics 23, 1846-1847. doi: 10.1093/bioinformatics/btm254

Esquela-Kerscher, A., and Slack, F. J. (2006). Oncomirs - microRNAs with a role in cancer. Nat. Rev. Cancer 6, 259-269. doi: 10.1038/nrc1840

Forrest, A. R. R., Kanamori-Katayama, M., Tomaru, Y., Lassmann, T., Ninomiya, N., Takahashi, Y., et al. (2010). Induction of microRNAs, mir-155, mir-222, mir-424 and mir-503, promotes monocytic differentiation through combinatorial regulation. Leukemia 24, 460-466. doi: 10.1038/leu. 2009.246 and COLIA1 (Stains et al., 2003; Stains and Civitelli, 2005b). The loss of GJA1 gap junctions diminishes ERK activity resulting in preferential recruitment of SP3 repressor to osteocalcin and COLIA1 gene promoters (Stains et al., 2003; Stains and Civitelli, 2005b). Here, we show that miR-23a gene targets in HOS cells are enriched for SP1 binding site within $2 \mathrm{~kb}$ of their transcription start site, which suggests that miR-23a may function by counteracting the effects of the SP1 family of transcription factors.

\section{Author Contributions}

YG and YJ wrote the manuscript. YJ, PF, and RW performed experiments. YG and YZ analyzed data. PM and PF conceived the study. PM supervised the work and edited the manuscript.

\section{Acknowledgments}

This study was funded by the Intramural Research Program of the National Cancer Institute.

\section{Supplementary Material}

The Supplementary Material for this article can be found online at: http://journal.frontiersin.org/article/10.3389/fgene. 2015.00233
Franceschi, R. T., Iyer, B. S., and Cui, Y. (1994). Effects of ascorbic acid on collagen matrix formation and osteoblast differentiation in murine MC3T3-E1 cells. J. Bone Min. Res. 9, 843-854. doi: 10.1002/jbmr. 5650090610

Friedman, R. C., Farh, K. K.-H., Burge, C. B., and Bartel, D. P. (2009). Most mammalian mRNAs are conserved targets of microRNAs. Genome Res. 19, 92-105. doi: 10.1101 /gr.082701.108

Hassan, M. Q., Gordon, J. A. R., Beloti, M. M., Croce, C. M., van Wijnen, A. J., Stein, J. L., et al. (2010). A network connecting Runx2, SATB2, and the miR23a 27a 24-2 cluster regulates the osteoblast differentiation program. Proc. Natl. Acad. Sci. U.S.A. 107, 19879-19884. doi: 10.1073/pnas.1007698107

Hassan, M. Q., Maeda, Y., Taipaleenmaki, H., Zhang, W., Jafferji, M., Gordon, J. A. R., et al. (2012). miR-218 Directs a Wnt signaling circuit to promote differentiation of osteoblasts and osteomimicry of metastatic cancer cells. J. Biol. Chem. 287, 42084-42092. doi: 10.1074/jbc.m112.377515

Hassan, M. Q., Tare, R. S., Lee, S. H., Mandeville, M., Morasso, M. I., Javed, A., et al. (2006). BMP2 commitment to the osteogenic lineage involves activation of Runx 2 by DLX3 and a homeodomain transcriptional network. J. Biol. Chem. 281, 40515-40526. doi: 10.1074/jbc.M604508200

Helman, L. J., and Meltzer, P. (2003). Mechanisms of sarcoma development. Nat. Rev. Cancer 3, 685-694. doi: 10.1038/nrc1168

Inose, H., Ochi, H., Kimura, A., Fujita, K., Xu, R., Sato, S., et al. (2009). A microRNA regulatory mechanism of osteoblast differentiation. Proc. Natl. Acad. Sci. U.S.A. 106, 20794-20799. doi: 10.1073/pnas.0909311106

Jiang, J. X., Siller-Jackson, A. J., and Burra, S. (2007). Roles of gap junctions and hemichannels in bone cell functions and in signal transmission of mechanical stress. Front. Biosci. 12, 1450-1462. doi: 10.2741/2159

Jones, K. B., Salah, Z., Del Mare, S., Galasso, M., Gaudio, E., Nuovo, G. J., et al. (2012). miRNA signatures associate with pathogenesis and progression of osteosarcoma. Cancer Res. 72, 1865-1877. doi: 10.1158/0008-5472.CAN-112663

Kobayashi, T., Lu, J., Cobb, B. S., Rodda, S. J., McMahon, A. P., Schipani, E., et al. (2008). Dicer-dependent pathways regulate chondrocyte proliferation 
and differentiation. Proc. Natl. Acad. Sci. U.S.A. 105, 1949-1954. doi: 10.1073/pnas.0707900105

Lecanda, F., Towler, D. A., Ziambaras, K., Cheng, S. L., Koval, M., Steinberg, T. H., et al. (1998). Gap junctional communication modulates gene expression in osteoblastic cells. Mol. Biol. Cell 9, 2249-2258. doi: 10.1091/mbc.9.8.2249

Lewis, B. P., Burge, C. B., and Bartel, D. P. (2005). Conserved seed pairing, often flanked by adenosines, indicates that thousands of human genes are microRNA targets. Cell 120, 15-20. doi: 10.1016/j.cell.2004.12.035

Li, Z., Hassan, M. Q., Volinia, S., van Wijnen, A. J., Stein, J. L., Croce, C. M., et al. (2008). A microRNA signature for a BMP2-induced osteoblast lineage commitment program. Proc. Natl. Acad. Sci. U.S.A. 105, 13906-13911. doi: $10.1073 /$ pnas. 0804438105

Livak, K. J., and Schmittgen, T. D. (2001). Analysis of relative gene expression data using real-time quantitative PCR and the 2(-Delta Delta C(T)) Method. Methods 25, 402-408. doi: 10.1006/meth.2001.1262

Logothetis, C. J., and Lin, S.-H. (2005). Osteoblasts in prostate cancer metastasis to bone. Nat. Rev. Cancer 5, 21-28. doi: 10.1038/nrc1528

Loiselle, A. E., Jiang, J. X., and Donahue, H. J. (2013). Gap junction and hemichannel functions in osteocytes. Bone 54, 205-212. doi: 10.1016/j.bone.2012.08.132

Longhi, A., Errani, C., De Paolis, M., Mercuri, M., and Bacci, G. (2006). Primary bone osteosarcoma in the pediatric age: State of the art. Cancer Treat. Rev. 32, 423-436. doi: 10.1016/j.ctrv.2006.05.005

Loots, G. G., Ovcharenko, I., Pachter, L., Dubchak, I., and Rubin, E. M. (2002). rVista for comparative sequence-based discovery of functional transcription factor binding sites. Genome Res. 12, 832-839. doi: 10.1101/gr.225502

Lu, J., Getz, G., Miska, E. A., Alvarez-Saavedra, E., Lamb, J., Peck, D., et al. (2005). MicroRNA expression profiles classify human cancers. Nature 435, 834-838. doi: 10.1038 /nature 03702

Lulla, R. R., Costa, F. F., Bischof, J. M., Chou, P. M., de F Bonaldo, M., Vanin, E. F., et al. (2011). Identification of differentially expressed microRNAs in osteosarcoma. Sarcoma 2011:732690. doi: 10.1155/2011/732690

Maire, G., Martin, J. W., Yoshimoto, M., Chilton-MacNeill, S., Zielenska, M., and Squire, J. A. (2011). Analysis of miRNA-gene expression-genomic profiles reveals complex mechanisms of microRNA deregulation in osteosarcoma. Cancer Genet. 204, 138-146. doi: 10.1016/j.cancergen.2010.12.012

Martin, J. W., Squire, J. A., and Zielenska, M. (2012). The genetics of osteosarcoma. Sarcoma 2012:627254. doi: 10.1155/2012/627254

Miao, J., Wu, S., Peng, Z., Tania, M., and Zhang, C. (2013). MicroRNAs in osteosarcoma: diagnostic and therapeutic aspects. Tumour Biol. 34, 2093-2098. doi: 10.1007/s13277-013-0940-7

Mirabello, L., Troisi, R. J., and Savage, S. A. (2009). Osteosarcoma incidence and survival rates from 1973 to 2004: data from the surveillance, epidemiology, and end results program. Cancer 115, 1531-1543. doi: 10.1002/cncr.24121

Nabavi, N., Pustylnik, S., and Harrison, R. E. (2012). Rab GTPase mediated procollagen trafficking in ascorbic acid stimulated osteoblasts. PLOS ONE 7:e46265. doi: 10.1371/journal.pone.0046265

Nakashima, K., Zhou, X., Kunkel, G., Zhang, Z., Deng, J. M., Behringer, R. R., et al. (2002). The novel zinc finger-containing transcription factor osterix is required for osteoblast differentiation and bone formation. Cell 108, 17-29. doi: 10.1016/S0092-8674(01)00622-5

Nuytten, M., Beke, L., Van Eynde, A., Ceulemans, H., Beullens, M., Van Hummelen, P., et al. (2008). The transcriptional repressor NIPP1 is an essential player in EZH2-mediated gene silencing. Oncogene 27, 1449-1460. doi: 10.1038/sj.onc.1210774

Plotkin, L. I., and Bellido, T. (2013). Beyond gap junctions: Connexin43 and bone cell signaling. Bone 52, 157-166. doi: 10.1016/j.bone.2012.09.030

PosthumaDeBoer, J., Witlox, M. A., Kaspers, G. J. L., and van Royen, B. J. (2011). Molecular alterations as target for therapy in metastatic osteosarcoma: a review of literature. Clin. Exp. Metast. 28, 493-503. doi: 10.1007/s10585-011-9384-x

Pustylnik, S., Fiorino, C., Nabavi, N., Zappitelli, T., da Silva, R., Aubin, J. E., et al. (2013). EB1 levels are elevated in ascorbic Acid (AA)-stimulated osteoblasts and mediate cell-cell adhesion-induced osteoblast differentiation. J. Biol. Chem. 288, 22096-22110. doi: 10.1074/jbc.M113.481515

Schiller, P. C., D'Ippolito, G., Balkan, W., Roos, B. A., and Howard, G. A. (2001). Gap-junctional communication is required for the maturation process of osteoblastic cells in culture. Bone 28, 362-369. doi: 10.1016/S87563282(00)00458-0

Siggelkow, H., Niedhart, C., Kurre, W., Ihbe, A., Schulz, A., Atkinson, M. J., et al. (1998). In vitro differentiation potential of a new human osteosarcoma cell line (HOS 58). Differ. Res. Biol. Divers. 63, 81-91. doi: 10.1046/j.14320436.1998.6320081.x

Simon, J. A., and Lange, C. A. (2008). Roles of the EZH2 histone methyltransferase in cancer epigenetics. Mutat. Res. 647, 21-29. doi: 10.1016/j.mrfmmm.2008.0 7.010

Smyth, G. K. (2005). Limma: Linear Models for Microarray Data. New York, NY: Springer.

Stains, J. P., and Civitelli, R. (2005a). Gap junctions in skeletal development and function. Biochim. Biophys. Acta 1719, 69-81. doi: 10.1016/j.bbamem.2005.1 0.012

Stains, J. P., and Civitelli, R. (2005b). Gap junctions regulate extracellular signalregulated kinase signaling to affect gene transcription. Mol. Biol. Cell 16, 64-72. doi: 10.1091/mbc.E04-04-0339

Stains, J. P., Lecanda, F., Screen, J., Towler, D. A., and Civitelli, R. (2003). Gap junctional communication modulates gene transcription by altering the recruitment of Sp1 and $\mathrm{Sp} 3$ to connexin-response elements in osteoblast promoters. J. Biol. Chem. 278, 24377-24387. doi: 10.1074/jbc.M212554200

Subramanian, A., Tamayo, P., Mootha, V. K., Mukherjee, S., Ebert, B. L., Gillette, M. A., et al. (2005). Gene set enrichment analysis: a knowledge-based approach for interpreting genome-wide expression profiles. Proc. Natl. Acad. Sci. U.S.A. 102, 15545-15550. doi: 10.1073/pnas.0506580102

Sugatani, T., and Hruska, K. A. (2007). MicroRNA-223 is a key factor in osteoclast differentiation. J. Cell. Biochem. 101, 996-999. doi: 10.1002/jcb.21335

Tang, N., Song, W.-X., Luo, J., Haydon, R. C., and He, T.-C. (2008). Osteosarcoma development and stem cell differentiation. Clin. Orthop. Relat. Res. 466, 2114-2130. doi: 10.1007/s11999-008-0335-z

Thomas, D., and Kansara, M. (2006). Epigenetic modifications in osteogenic differentiation and transformation. J. Cell. Biochem. 98, 757-769. doi: $10.1002 / \mathrm{jcb} .20850$

Wang, X., Dalkic, E., Wu, M., and Chan, C. (2008). Gene module level analysis: identification to networks and dynamics. Curr. Opin. Biotechnol. 19, 482-491. doi: 10.1016/j.copbio.2008.07.011

Wei, Y., Chen, Y.-H., Li, L.-Y., Lang, J., Yeh, S.-P., Shi, B., et al. (2011). CDK1dependent phosphorylation of EZH2 suppresses methylation of H3K27 and promotes osteogenic differentiation of human mesenchymal stem cells. Nat. Cell Biol. 13, 87-94. doi: 10.1038/ncb2139

Yi, R., Poy, M. N., Stoffel, M., and Fuchs, E. (2008). A skin microRNA promotes differentiation by 1 repressing 'stemness'. Nature 452, 225-229. doi: 10.1038 /nature06642

Zhang, Y., Xie, R.-L., Croce, C. M., Stein, J. L., Lian, J. B., van Wijnen, A. J., et al. (2011). A program of microRNAs controls osteogenic lineage progression by targeting transcription factor Runx2. Proc. Natl. Acad. Sci. U.S.A. 108, 9863-9868. doi: 10.1073/pnas.1018493108

Zhang, Y., Xie, R.-L., Gordon, J., LeBlanc, K., Stein, J. L., Lian, J. B., et al. (2012). Control of mesenchymal lineage progression by microRNAs targeting skeletal gene regulators Trps1 and Runx2. J. Biol. Chem. 287, 21926-21935. doi: 10.1074/jbc.M112.340398

Zhou, G., Shi, X., Zhang, J., Wu, S., and Zhao, J. (2013). MicroRNAs in osteosarcoma: from biological players to clinical contributors, a review. J. Int. Med. Res. 41, 1-12. doi: 10.1177/0300060513475959

Conflict of Interest Statement: The authors declare that the research was conducted in the absence of any commercial or financial relationships that could be construed as a potential conflict of interest.

Copyright (๑) 2015 Gindin, Jiang, Francis, Walker, Abaan, Zhu and Meltzer. This is an open-access article distributed under the terms of the Creative Commons Attribution License (CC BY). The use, distribution or reproduction in other forums is permitted, provided the original author(s) or licensor are credited and that the original publication in this journal is cited, in accordance with accepted academic practice. No use, distribution or reproduction is permitted which does not comply with these terms. 